JOURNAL OF

FUNCTION SPACES AND APPLICATIONS

Volume 5, Number 2 (2007), 183-198 (c) 2007, Scientific Horizon

http://www.jfsa.net

\title{
A direct proof of Sobolev embeddings for quasi-homogeneous Lizorkin-Triebel spaces with mixed norms
}

\author{
Jon Johnsen and Winfried Sickel
}

(Communicated by Jürgen Appell)

2000 Mathematics Subject Classification. 46E35.

Keywords and phrases. Function spaces of Besov and Lizorkin-Triebel type, anisotropic spaces, mixed norms, Sobolev embeddings, geometric rectangle condition.

\footnotetext{
Abstract. The article deals with a simplified proof of the Sobolev embedding theorem for Lizorkin-Triebel spaces (that contain the $L_{p}$-Sobolev spaces $H_{p}^{s}$ as special cases). The method extends to a proof of the corresponding fact for general Lizorkin-Triebel spaces based on mixed $L_{p}$-norms. In this context a Nikol'skij-Plancherel-Polya inequality for sequences of functions satisfying a geometric rectangle condition is proved. The results extend also to anisotropic spaces of the quasi-homogeneous type.
}

\section{Introduction}

To give an overview, we first comment on standard Lizorkin-Triebel spaces (i.e. isotropic, inhomogeneous spaces with unmixed norms). These are throughout denoted by $F_{p, q}^{s}$.

Since around 1977 the question of Sobolev embeddings of Lizorkin-Triebel spaces has been answered affirmatively, with a unified proof of the following 
Proposition 1. Let $s, s_{0} \in \mathbb{R}$ and $\left.p, p_{0} \in\right] 0, \infty[$ be given such that

$$
s-\frac{n}{p}=s_{0}-\frac{n}{p_{0}}, \quad p>p_{0} .
$$

There is then a continuous embedding $F_{p_{0}, \infty}^{s_{0}}\left(\mathbb{R}^{n}\right) \hookrightarrow F_{p, q}^{s}\left(\mathbb{R}^{n}\right)$ for every $q \in$ ] $0, \infty]$.

Specifically this means that there exists a number $C>0$, depending on the parameters, such that the following inequality is valid for every $u \in F_{p_{0}, \infty}^{s_{0}}\left(\mathbb{R}^{n}\right)$ :

$$
\left(\int_{\mathbb{R}^{n}}\left(\sum_{j=0}^{\infty} 2^{s j q}\left|u_{j}(x)\right|^{q}\right)^{\frac{p}{q}} d x\right)^{\frac{1}{p}} \leq C\left(\int_{\mathbb{R}^{n}}\left(\sup _{j \in \mathbb{N}_{0}} 2^{s_{0} j}\left|u_{j}(x)\right|\right)^{p_{0}} d x\right)^{\frac{1}{p_{0}}}
$$

To explain the notation, note that a Littlewood-Paley decomposition $1=$ $\sum_{j=0}^{\infty} \Phi_{j}(\xi)$ can be obtained by letting $\Phi_{j}=\Psi_{j}-\Psi_{j-1}$ for $j>0$ when $\Psi_{j}(\xi)=\psi\left(2^{-j}|\xi|\right)$ for some $\psi \in C^{\infty}(\mathbb{R}, \mathbb{R})$ fulfilling $\psi(t)=1$ for $t<1$ and $\psi(t)=0$ for $t>2$.

By definition, a tempered distribution $u \in \mathcal{S}^{\prime}\left(\mathbb{R}^{n}\right)$ is in the Lizorkin-Triebel space $F_{p, q}^{s}\left(\mathbb{R}^{n}\right)$ when the quantity on the left hand side of (2) is finite; hereby $u_{j}=\mathcal{F}^{-1}\left(\Phi_{j} \hat{u}\right)$. As usual $\|f\|_{p}$ denotes the norm of a function $f$ in $L_{p}\left(\mathbb{R}^{n}\right)$ with respect to the Lebesgue measure $\lambda$ on $\mathbb{R}^{n}$.

Jawerth's original proof [8] of the Sobolev inequalities (2) was given for the homogeneous spaces $\dot{F}_{p, q}^{s}$, utilising the rewriting of $L_{p}$-norms via the distribution function $(0<p<\infty)$

$$
\int_{\mathbb{R}^{n}}|f(x)|^{p} d x=p \int_{0}^{\infty} t^{p-1} \lambda(\{x|| f(x) \mid>t\}) d t .
$$

However, readers familiar with the subject may recall that for the inhomogeneous $F_{p, q}^{s}$, it is not possible for every $t>0$ to find $j \in \mathbb{N}_{0}$ such that $2^{j n / p}<t$ (unlike $\dot{F}_{p, q}^{s}$, where $j \in \mathbb{Z}$ ), so Jawerth's proof needs an adaptation to this case. Triebel $[17,18]$ introduced a splitting into the $t$-intervals $] 0, K[$ and $] K, \infty[$, where $K$ essentially was the $q^{\text {th }}$ root of $\left\|u \mid F_{p, q}^{s}\right\|$.

Since then this proof has widely been considered 'best possible'. Perhaps this is because the strategy of Jawerth and Triebel covers all $p \in] 0, \infty[$ in an elegant way (at least for $\dot{F}_{p, q}^{s}$ ), whilst previous attempts did not cover all cases.

In comparison, the Besov spaces $B_{p, q}^{s}\left(\mathbb{R}^{n}\right)$ have corresponding embeddings with a well-known one-line proof based on the Nikol' skij-Plancherel-Polya inequality that we now recall.

For $0<p \leq r \leq \infty$ there exists a $c>0$ such that for every $f \in \mathcal{S}^{\prime}\left(\mathbb{R}^{n}\right) \cap L_{p}$ with supp $\mathcal{F} f$ compact, say contained in the closed ball $B(0, R)=\left\{\xi \in \mathbb{R}^{n} \mid\right.$ $|\xi| \leq R\}$

$$
\|f\|_{r} \leq c R^{\frac{n}{p}-\frac{n}{r}}\|f\|_{p} .
$$


Applying this to each $u_{j}\left(R=2^{j+1}\right)$ in $\left\|u \mid B_{p, q}^{s}\right\|:=\left(\sum 2^{s j q}\left\|u_{j}\right\|_{p}^{q}\right)^{\frac{1}{q}}$, one finds at once that $B_{p_{0}, q}^{s_{0}} \hookrightarrow B_{p, q}^{s}$ when (1) holds.

Generally $F_{p, q}^{s}$-spaces are rather more complicated to treat than $B_{p, q}^{s}$-spaces, so it would seem plausible that even the basic Sobolev inequalities in (2) are substantially more technical to achieve for the $F_{p, q}^{s}$.

But also (2) has a short proof based on the Nikol'skij-Plancherel-Polya inequality (4). The trick is to handle the infinite sum by means of the following result on the weighted sequence spaces $\ell_{q}^{s}$, that (for $q<\infty$ ) is normed by

$$
\left\|a_{j} \mid \ell_{q}^{s}\right\|=\left(\sum_{j=0}^{\infty} 2^{s j q}\left|a_{j}\right|^{q}\right)^{\frac{1}{q}} .
$$

Lemma 2. Let real numbers $s_{1}<s_{0}$ be given, and $\left.\theta \in\right] 0,1[$. For $0<q \leq \infty$ there is $c>0$ such that

$$
\left\|a_{j}\left|\ell_{q}^{\theta s_{0}+(1-\theta) s_{1}}\|\leq c\| a_{j}\right| \ell_{\infty}^{s_{0}}\right\|^{\theta}\left\|a_{j} \mid \ell_{\infty}^{s_{1}}\right\|^{1-\theta}
$$

holds for all complex sequences $\left(a_{j}\right)_{j \in \mathbb{N}_{0}}$ in $\ell_{\infty}^{s_{0}}$.

This result was to our knowledge first crystallised in the works of Brezis and Mironescu [3]; cf. the elementary proof there.

In what follows we shall discuss certain generalizations of Proposition 1, and of (4), to mixed $L_{p}$-norms and anisotropic smoothness. For the reader's convenience we shall first give the details of the proof in the isotropic (unmixed) case. Hence we prove Proposition 1; as it will become clear in Sections 2 and 3, the method carries over straightforwardly to the more general situation.

Proof. [Proof of Proposition 1 (based on (4), (6))] For $u \in F_{p_{0}, \infty}^{s_{0}}$ the claim is obtained by interpolating its $F_{p, q}^{s}$-norm between those of $B_{\infty, \infty}^{s_{1}, \infty}$ with $s_{1}:=s_{0}-\frac{n}{p_{0}}=s-\frac{n}{p}$ and the given space $F_{p_{0}, \infty}^{s_{0}}$ :

Since $p>p_{0}$ there is some $\left.\theta \in\right] 0,1\left[\right.$ so that $\theta \frac{n}{p_{0}}=\frac{n}{p}$, hence

$$
\theta s_{0}+(1-\theta) s_{1}=\theta s_{0}+(1-\theta)\left(s_{0}-\frac{n}{p_{0}}\right)=s-\frac{n}{p}+\theta \frac{n}{p_{0}}=s .
$$

Clearly $q<\infty$ suffices in (2), and for each $x \in \mathbb{R}^{n}$, Lemma 2 gives

$$
\left(\sum_{j=0}^{\infty} 2^{s j q}\left|u_{j}(x)\right|^{q}\right)^{\frac{1}{q}} \leq c\left\|u_{j}(x) \mid \ell_{\infty}^{s_{0}}\right\|^{\theta}\left(\sup _{j} 2^{s_{1} j}\left\|u_{j}\right\|_{\infty}\right)^{1-\theta} .
$$

Here $\left\|u_{j}\right\|_{\infty} \leq c^{\prime} 2^{j \frac{n}{p_{0}}}\left\|u_{j}\right\|_{p_{0}}$ follows from (4) since $u_{j}$ is in $L_{p_{0}}$ with supp $\mathcal{F} u_{j} \subset$ $B\left(0,2^{j+1}\right)$. The definition of $s_{1}$ therefore yields

$$
\sup _{j} 2^{s_{1} j}\left\|u_{j}\right\|_{\infty} \leq c^{\prime} \sup _{j} 2^{\left(s_{1}+\frac{n}{p_{0}}\right) j}\left\|u_{j}\right\|_{p_{0}} \leq c^{\prime}\left\|u \mid F_{p_{0}, \infty}^{s_{0}}\right\| .
$$


Hence the fact that $\theta p=p_{0}$ gives, by taking $L_{p}$-norms in (8),

$$
\left\|u\left|F_{p, q}^{s}\|\leq c\| u\right| F_{p_{0}, \infty}^{s_{0}}\right\|^{\theta}\left(c^{\prime}\left\|u \mid F_{p_{0}, \infty}^{s_{0}}\right\|\right)^{1-\theta}=c^{\prime \prime}\left\|u \mid F_{p_{0}, \infty}^{s_{0}}\right\| .
$$

That is, (2) is proved.

Remark 3. For simplicity we have taken the sum-exponent $q_{0}=\infty$ on the right hand side of (2), but the other Sobolev inequalities can be recovered via the simple embedding $F_{p_{0}, q_{0}}^{s_{0}} \hookrightarrow F_{p_{0}, \infty}^{s_{0}}$.

The Nikol'skij-Plancherel-Polya inequality (4) will be generalised to mixed $L_{p}$-norms in Section 2 below. In the course of the proof given there, we utilise (4) in the above unmixed form; so to provide a complete overview we insert an argument for this (known) standard version.

Proof. [Proof of inequality (4)] It suffices to establish (4) when in addition to the stated assumptions $u \in \mathcal{S}\left(\mathbb{R}^{n}\right)$, where $\mathcal{S}\left(\mathbb{R}^{n}\right)$ is the Schwartz space of rapidly decreasing $C^{\infty}$-functions. To see this we take $\hat{\psi} \in C^{\infty}\left(\mathbb{R}^{n}\right)$ such that $\hat{\psi}(t)=1$ for $|t| \leq 1, \hat{\psi}(t)=0$ for $|t| \geq 2$, while $\psi(0)=1$. Defining $u_{j}$ by

$$
u_{j}(x):=u(x) \psi\left(\frac{x}{j}\right)
$$

then $u_{j}$ has its spectrum in $\operatorname{supp} \hat{u}+B\left(0, \frac{2}{j}\right)$, and $u_{j} \in \mathcal{S}\left(\mathbb{R}^{n}\right)$ for $u(x)$ is $\mathcal{O}\left((1+|x|)^{N}\right)$ for some $N \geq 0$ by the Paley-Wiener-Schwartz theorem $\left(\hat{u} \in \mathcal{E}^{\prime}\right)$. There is only something to show for $p<r$, so $p<\infty$ and hence

$$
u_{j} \rightarrow u \quad \text { in } \mathcal{S}^{\prime}\left(\mathbb{R}^{n}\right) \cap L_{p}\left(\mathbb{R}^{n}\right)
$$

So if (4) can be proved for all Schwartz functions with compact spectra, it will follow that $\left(u_{j}\right)$ is a fundamental sequence in $L_{r}$, hence converging to some $g \in L_{r}$ with $g(x)=u(x)$ a.e.

Since $\|\cdot\|_{p}$ is continuous $L_{p} \rightarrow \mathbb{R}$, the above would entail that

$$
\left\|u\left|L_{r}\left\|\leq \lim _{j \rightarrow \infty} c\left(R+\frac{2}{j}\right)^{\frac{n}{p}-\frac{n}{r}}\right\| u_{j}\right| L_{p}\right\|=c R^{\frac{n}{p}-\frac{n}{r}}\left\|u \mid L_{p}\right\| .
$$

( $c>0$ will be independent of $u$ and the size of its spectrum.)

In the smooth case we proceed in the spirit of [18, 1.3.2]. When $u \in \mathcal{S}\left(\mathbb{R}^{n}\right)$ with $\hat{u} \in C_{B(0, R)}^{\infty}$, then $\Psi(x)=R^{n} \psi(R x)$ fulfils

$$
u(x)=\mathcal{F}^{-1}(\hat{\Psi} \hat{u})=\int_{\mathbb{R}^{n}} u(y) \Psi(x-y) d y
$$

For $1 \leq p \leq \infty=r$ and $p+p^{*}=p p^{*}$, the above identity gives

$$
|u(x)| \leq\left\|\Psi\left|L_{p^{*}}\|\| u\right| L_{p}\right\| \leq\left\|\psi\left|L_{p^{*}}\left\|R^{\frac{n}{p}}\right\| u\right| L_{p}\right\|,
$$


hence (4). In case $0<p<1$ and $r=\infty$,

$$
|u(x)| \leq\|\Psi\|_{\infty}(\sup |u(z)|)^{1-p} \int|u(y)|^{p} d y,
$$

so the $x$-independence of the right hand side entails

$$
|u(x)|^{p} \leq\|u\|_{\infty}^{p} \leq\|\Psi\|_{\infty} \int|u(y)|^{p} d y
$$

where $\|\Psi\|_{\infty}^{\frac{1}{p}}=R^{\frac{n}{p}}\|\psi\|_{\infty}$. This proves (4) for $r=\infty$.

If $r<\infty$ and $p<1$, insertion of (17) into $\|u\|_{r}$ gives

$$
\begin{aligned}
\left(\int|u(x)|^{r} d x\right)^{\frac{1}{r}} & \left.\leq \sup _{z}|u(z)|^{r-p}\right)^{\frac{1}{r}}\left(\int|u(x)|^{p} d x\right)^{\frac{1}{r}} \\
& \leq\|\Psi\|_{\infty}^{\frac{r-p}{r p}}\|u\|_{p} \leq\left(\|\psi\|_{\infty} R^{n}\right)^{\frac{1}{p}-\frac{1}{r}}\|u\|_{p} .
\end{aligned}
$$

For $1 \leq p \leq r<\infty$ one can insert (15) instead. This completes the proof of (4), with $c$ equal to a power of a norm of $\psi$.

\section{The mixed norm case}

One advantage of the above proof method is that it extends to spaces with mixed norms. In this set-up $L_{p}\left(\mathbb{R}^{n}\right)$ is replaced by $L_{\vec{p}}\left(\mathbb{R}^{n}\right)$ where $\vec{p}=\left(p_{1}, \ldots, p_{n}\right)$ for $0<p_{j} \leq \infty, j=1, \ldots, n$, and

$$
\left\|f \mid L_{\vec{p}}\right\|=\left(\int_{\mathbb{R}}\left(\ldots\left(\int_{\mathbb{R}}\left|f\left(x_{1}, \ldots, x_{n}\right)\right|^{p_{1}} d x_{1}\right)^{\frac{p_{2}}{p_{1}}} \ldots\right)^{\frac{p_{n}}{p_{n}-1}} d x_{n}\right)^{\frac{1}{p_{n}}} .
$$

It is well known that such spaces frequently enter the analysis of evolution problems for partial differential equations.

The corresponding Lizorkin-Triebel spaces $F_{\vec{p}, q}^{s}\left(\mathbb{R}^{n}\right)$ consist of the $u$ in $\mathcal{S}^{\prime}\left(\mathbb{R}^{n}\right)$ having finite quasi-norms $\left(0<p_{j}<\infty\right)$

$$
\left\|u\left|F_{\vec{p}, q}^{s}\|=\|\left(\sum_{j=0}^{\infty} 2^{s q j}\left|u_{j}(\cdot)\right|^{q}\right)^{\frac{1}{q}}\right| L_{\vec{p}}\right\| .
$$

The purpose is not to go into the general theory of such spaces here. Instead we want to show that the Sobolev embeddings follow directly from first principles,

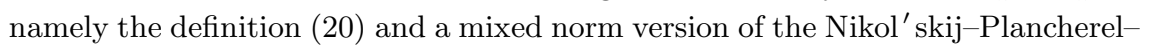
Polya inequality. 
Before we turn to this, it is noted that also the mixed quasi-norm in $L_{\vec{p}}$ is subadditive when raised to a power $\left.\lambda \in] 0, \min \left(1, p_{1}, \ldots, p_{n}\right)\right]$,

$$
\left\|f+g\left|L_{\vec{p}}\left\|^{\lambda} \leq\right\| f\right| L_{\vec{p}}\right\|^{\lambda}+\left\|g \mid L_{\vec{p}}\right\|^{\lambda} .
$$

In fact $\int|f+g|^{p} d \mu \leq \int|f|^{p} d \mu+\int|g|^{p} d \mu$ holds for arbitrary measures if $0<p \leq 1$, so $\|f+g\|_{p} \leq\left(\|f\|_{p}^{\nu}+\|g\|_{p}^{\nu}\right)^{1 / \nu}$ for all $\left.\left.\nu \in\right] 0, \min (1, p)\right]$. Using this, it is easy to see that \|\|$\cdot\left\|_{q}\right\|_{p}^{\lambda}$ is subadditive for $\lambda \leq \min (1, p, q)$. For $\lambda=\min \left(1, p_{1}, \ldots, p_{n}\right)$ a repeated use of this yields $(21)$.

To prepare for the mixed norm version of the Nikol'skij-Plancherel-Polya inequality, we recall the Paley-Wiener-Schwartz Theorem in a precise version, cf. [7, Ch. 7]: when $K \Subset \mathbb{R}^{n}$, a distribution $u \in \mathcal{S}^{\prime}\left(\mathbb{R}^{n}\right)$ fulfils $\operatorname{supp} \hat{u} \subset K$ if and only if $u$ extends to a function $u(x+\mathrm{i} y)$ on $\mathbb{C}^{n}$ which is entire analytic and fulfils

$$
|u(x+\mathrm{i} y)| \leq C(1+|x+\mathrm{i} y|)^{N} e^{H(-y)}, \quad x, y \in \mathbb{R}^{n}
$$

for some $N \geq 0$ (the order of $\hat{u}$ ); here $H(y)=\sup \{y \cdot \xi \mid \xi \in K\}$ is the supporting function of the compact set $K$.

When $u \in \mathcal{S}^{\prime} \cap L_{\vec{p}}\left(\mathbb{R}^{n}\right)$ and supp $\hat{u} \Subset \mathbb{R}^{n}$, then $u(x)=\mathcal{O}\left((1+|x|)^{N}\right)$ for $x \in \mathbb{R}^{n}$, so $u \in L_{1}^{\text {loc }}\left(\mathbb{R}^{n}\right)$ also if some $p_{k}<1$; and (22) also yields $u(\varphi)=\int u(x) \varphi(x) d x$ for $\varphi \in \mathcal{S}\left(\mathbb{R}^{n}\right)$. (In general elements of $\mathcal{S}^{\prime} \cap L_{1}^{\text {loc }}$ do not act on $\mathcal{S}$ by integration; cf. $e^{x} \cos \left(e^{x}\right)=\sin \left(e^{x}\right)^{\prime}$ on $\mathbb{R}$.)

We also note that for a rectangle $\left[-R_{1}, R_{1}\right] \times \cdots \times\left[R_{n}, R_{n}\right]$,

$$
H(y)=R_{1}\left|y_{1}\right|+\cdots+R_{n}\left|y_{n}\right|
$$

Indeed, $H(y)$ can be estimated by the triangle inequality, and equality is attained in one of the corners $\left( \pm R_{1}, \ldots, \pm R_{n}\right)$. When $\hat{u}$ is supported in this rectangle, and $\left(x^{\prime}, x^{\prime \prime}\right),\left(y^{\prime}, y^{\prime \prime}\right) \in \mathbb{R}^{n^{\prime}} \times \mathbb{R}^{n^{\prime \prime}}, n=n^{\prime}+n^{\prime \prime}$, it follows when $x^{\prime \prime}, y^{\prime \prime}$ are kept as parameters that $U\left(x^{\prime}+\mathrm{i} y^{\prime}\right):=u\left(\left(x^{\prime}, x^{\prime \prime}\right)+\mathrm{i}\left(y^{\prime}, y^{\prime \prime}\right)\right)$ is analytic on $\mathbb{C}^{n^{\prime}}$ and

$$
\left|U\left(x^{\prime}+\mathrm{i} y^{\prime}\right)\right| \leq C^{\prime}\left(1+\left|\left(x^{\prime}, y^{\prime}\right)\right|\right)^{N} \exp \left(\sum_{n^{\prime}} R_{j}\left|y_{j}\right|\right) .
$$

Therefore $x^{\prime} \mapsto U\left(x^{\prime}\right)$ is a tempered distribution on $\mathbb{R}^{n^{\prime}}$ with spectrum in $\prod_{n^{\prime}}\left[-R_{j}, R_{j}\right]$, as one would expect.

These facts are convenient for the proof of

Proposition 4. Whenever $0<p_{j} \leq r_{j} \leq \infty$ for $j=1, \ldots, n$, there is a $c>0$ such that for every $f \in \mathcal{S}^{\prime}\left(\mathbb{R}^{n}\right) \cap L_{\vec{p}}$ with spectrum in a compact rectangle given by $\left|\xi_{k}\right| \leq R_{k}$ for $k=1, \ldots, n$, i.e.

$$
\operatorname{supp} \mathcal{F} f \subset\left[-R_{1}, R_{1}\right] \times \ldots\left[-R_{n}, R_{n}\right]
$$


it also holds that $f \in L_{\vec{r}}\left(\mathbb{R}^{n}\right)$ and

$$
\left\|f\left|L_{\vec{r}}\left\|\leq c\left(\prod_{k=1, \ldots, n} R_{k}^{\frac{1}{p_{k}}-\frac{1}{r_{k}}}\right)\right\| f\right| L_{\vec{p}}\right\| .
$$

This result was established by Unin'skij $[19,20]$ for exponents $p_{k} \geq 1$; Schmeisser and Triebel $[12,1.6 .2]$ covered the case $n=2$. We give a direct proof where the treatment of $0<p_{j}<1$ is inspired by a paper of Stöckert [14, Satz 2.1], who proved (26) for subclasses of $\mathcal{S}\left(\mathbb{R}^{n}\right)$ with exponential decay.

The strategy of the proof is perhaps best described as a succession of embeddings, which means that (26) is realised as a composition of the following $n$ embeddings, that each only affect a single coordinate direction:

$$
L_{\vec{p}} \hookrightarrow L_{\left(r_{1}, p_{2}, \ldots, p_{n}\right)} \hookrightarrow L_{\left(r_{1}, r_{2}, p_{3}, \ldots, p_{n}\right)} \hookrightarrow \ldots \hookrightarrow L_{\vec{r}} .
$$

For $r_{k}=p_{k}$ the $k^{\text {th }}$ map is just the identity map from the $k^{\text {th }}$ space to itself. This gives a convenient reduction to the case in which $\vec{r}$ and $\vec{p}$ differ in only one component, say $r_{m}>p_{m}$ whilst $r_{j}=p_{j}$ for $j \neq m$. Thereby some technicalities are circumvented. (Already (12) is troublesome to carry over to the mixed case, for if some $p_{k}=\infty$ we cannot obtain convergence in the norm topology.)

Proof. $1^{\circ}$. We prove (26) for an arbitrary $u \in L_{\vec{p}}\left(\mathbb{R}^{n}\right)$ by means of a succession of embeddings, as explained above. So it suffices to assume that $r_{m}>p_{m}$ only holds for one value of $m$, and we can assume that this is $m=n$.

Indeed, seeing $u$ as a function of $x^{\prime}=\left(x_{1}, \ldots, x_{m}\right)$, it was found in (24) ff. that its spectrum lies in $\left[-R_{1}, R_{1}\right] \times \cdots \times\left[-R_{m}, R_{m}\right]$; and by $(22)$ it is in $\mathcal{S}^{\prime} \cap L_{\left(p_{1}, \ldots, p_{m}\right)}$ on $\mathbb{R}^{m}$, so once the case $m=n$ is covered, integration with respect to $x^{\prime \prime}$ yields (26) in general when $1 \leq m \leq n$.

$2^{\circ}$. If $\psi \in \mathcal{S}(\mathbb{R})$ is such that $\hat{\psi}(t)=0$ for $|t| \geq 2$ while $\hat{\psi}(t)=1$ for $|t| \leq 1$, we set $\Psi\left(x_{n}\right)=R_{n} \psi\left(R_{n} x_{n}\right)$. Clearly $\hat{u}(\xi)=\hat{\Psi}\left(\xi_{n}\right) \hat{u}(\xi)$, and we want to show that

$$
u(x)=\int_{\mathbb{R}} u\left(x^{\prime}, y_{n}\right) \Psi\left(x_{n}-y_{n}\right) d y_{n} \quad \text { for } \quad x \in \mathbb{R}^{n} .
$$

The right hand side makes sense by (22), and for $\varphi \in C_{0}^{\infty}\left(\mathbb{R}^{n}\right)$

$$
\mathcal{F}_{x \rightarrow \xi}\left(\int \varphi\left(x^{\prime}, y_{n}\right) \Psi\left(x_{n}-y_{n}\right) d y_{n}\right)=\hat{\Psi}\left(\xi_{n}\right) \hat{\varphi}(\xi) .
$$

From this and the inversion formula $\mathcal{F}^{2} \varphi=(2 \pi)^{n} \varphi(-\cdot)$ used twice,

$$
\begin{aligned}
\langle u, \varphi(-\cdot)\rangle & =\langle\hat{u}, \hat{\varphi}\rangle /(2 \pi)^{n}=\left\langle\hat{u}, \hat{\Psi}\left(\xi_{n}\right) \hat{\varphi}(\xi)\right\rangle /(2 \pi)^{n} \\
& =\left\langle u, \mathcal{F}_{\xi \rightarrow x}\left(\hat{\Psi}\left(\xi_{n}\right) \hat{\varphi}(\xi)\right)\right\rangle /(2 \pi)^{n} \\
& =\iiint u(x) \Psi\left(y_{n}-x_{n}\right) \varphi\left(-x^{\prime},-y_{n}\right) d x_{n} d y_{n} d x^{\prime} .
\end{aligned}
$$


The last identity follows from Fubini's theorem and the polynomial growth. In particular $\left(x^{\prime}, y_{n}\right) \mapsto \int u(x) \Psi\left(y_{n}-x_{n}\right) d x_{n} \varphi\left(-x^{\prime},-y_{n}\right)$ is integrable, and since $u$ itself acts by integration, this shows (28) in the set of locally integrable functions.

$3^{\circ}$. In case $p_{k} \geq 1$ for all $k$ it follows from (28) and the generalised Minkowski inequality that

$$
\left\|u\left(\cdot, x_{n}\right)\left|L_{p^{\prime}}\left(\mathbb{R}^{n-1}\right)\left\|\leq \int\left|\Psi\left(x_{n}-y_{n}\right)\right| \cdot\right\| u\left(\cdot, y_{n}\right)\right| L_{p^{\prime}}\right\| d y_{n} .
$$

The Hausdorff-Young inequality applies to the convolution in the last expression, so if $q \geq 1$ is defined by $\frac{1}{p_{n}}+\frac{1}{q}=1+\frac{1}{r_{n}}$,

$$
\left\|u\left|L_{\vec{r}}\|\leq\| \Psi\right| L_{q}(\mathbb{R})\right\|\left\|u\left|L_{\vec{p}}\left\|\leq R_{n}^{1-\frac{1}{q}}\right\| \psi\left\|_{q}\right\| u\right| L_{\vec{p}}\right\|
$$

Because $1-\frac{1}{q}=\frac{1}{p_{n}}-\frac{1}{r_{n}}$, this yields (26) for all classical exponents.

$4^{\circ}$. In general there is some $\left.s \in\right] 0,1\left[\operatorname{such}\right.$ that $\frac{p_{k}}{s}>1$ for all $k$. With this (28) can be replaced by

$$
|u(x)|^{s} \leq C\left(R_{n}\right) \int_{\mathbb{R}}\left|u\left(x^{\prime}, y_{n}\right)\right|^{s}\left|\Psi\left(x_{n}-y_{n}\right)\right|^{s} d y_{n} .
$$

Indeed, $y_{n} \mapsto u\left(x^{\prime}, y_{n}\right) \Psi\left(x_{n}-y_{n}\right)$ has spectrum in $\left[-3 R_{n}, 3 R_{n}\right]$ (since that of $u\left(x^{\prime}, \cdot\right)$ is contained in $\left.\left[-R_{n}, R_{n}\right]\right)$. Hence (33) results from the next inequality, that follows from $2^{\circ}$ and the standard Nikol' skij-Plancherel-Polya inequality (4) on $\mathbb{R}$,

$$
|u(x)| \leq\left\|u\left(x^{\prime}, \cdot\right) \Psi\left(x_{n}-\cdot\right)\right\|_{1} \leq c\left(3 R_{n}\right)^{\frac{1}{s}-1}\left\|u\left(x^{\prime}, \cdot\right) \Psi\left(x_{n}-\cdot\right)\right\|_{s} .
$$

Applying the generalised Minkowski inequality to (33) we first obtain

$$
\left\|\left.\left|u\left(\cdot, x_{n}\right)\right|^{s}\left|L_{p^{\prime} / s}\left\|\leq C\left(R_{n}\right) \int\left|\Psi\left(x_{n}-y_{n}\right)\right|^{s}\right\|\right| u\left(\cdot, y_{n}\right)\right|^{s} \mid L_{p^{\prime} / s}\right\| d y_{n},
$$

so by taking $q \geq 1$ such that $1+\frac{1}{r_{n} / s}=\frac{1}{p_{n} / s}+\frac{1}{q / s}$,

$$
\left\|\left.\left.|u|^{s}\left|L_{\vec{r} / s}\left\|\leq C\left(R_{n}\right)\right\|\right| \Psi\right|^{s}\left|L_{q / s}\|\|\right| u\right|^{s} \mid L_{\vec{p} / s}\right\|
$$

But this means that

$$
\left\|u\left|L_{\vec{r}}\left\|^{s} \leq c^{\prime} R_{n}^{1-s} R_{n}^{s-\frac{s}{q}}\right\| \psi\left\|_{q}^{s}\right\| u\right| L_{\vec{p}}\right\|^{s}
$$

and since $1-\frac{s}{q}=s\left(\frac{1}{p_{n}}-\frac{1}{r_{n}}\right)$ the claim follows by taking roots.

Using Lemma 2 and ideas from the proof of Proposition 1, the above theorem can now relatively easily be extended to a sequence version of the Nikol'skijPlancherel-Polya inequality. 
This version deals with sequences $\left(f_{j}\right)$ in $\mathcal{S}^{\prime}\left(\mathbb{R}^{n}\right)$ that fulfill the following spectral condition, that we could describe as a geometric rectangle condition,

$$
\operatorname{supp} \mathcal{F} f_{j} \subset\left[-A R_{1}^{j}, A R_{1}^{j}\right] \times \cdots \times\left[-A R_{n}^{j}, A R_{n}^{j}\right]
$$

Here $A>0$ is a constant, while the fixed numbers $R_{1}, \ldots, R_{n}>1$ define the rectangles.

The next inequality will give the Sobolev embeddings in Theorem 6 below at once, but the inequality is also interesting for other purposes; it was obtained in [12, Prop. 2.4.1] for $n=2$.

Theorem 5. When $\vec{p} \neq \vec{r}$ and $0<p_{k} \leq r_{k}<\infty$ for $k=1, \ldots, n$, then there is for $0<q \leq \infty$ a number $c>0$ such that

$$
\left\|\left(\sum_{j=0}^{\infty}\left|f_{j}(\cdot)\right|^{q}\right)^{\frac{1}{q}}\left|L_{\vec{r}}\|\leq c\| \sup _{j \in \mathbb{N}_{0}}\left(\prod_{k=1}^{n} R_{k}^{\frac{j}{p_{k}}-\frac{j}{r_{k}}}\left|f_{j}(\cdot)\right|\right)\right| L_{\vec{p}}\right\|
$$

for all sequences $\left(f_{j}\right)$ in $\mathcal{S}^{\prime}\left(\mathbb{R}^{n}\right)$ fulfilling $(38)$.

Proof. Using a succession of embeddings as in the proof of Proposition 4, it is enough to cover the case in which $r_{m} \neq p_{m}$ only holds for $m=n$.

Furthermore it can be assumed that $q<\min \left(r_{1}, \ldots, r_{n}\right)$. Then since $r_{n}<\infty$, it is a consequence of Minkowski's inequality that the left hand side of (39) is less than

$$
\left(\int_{\mathbb{R}}\left(\sum_{j=0}^{\infty}\left\|f_{j}\left(\cdot, x_{n}\right) \mid L_{p^{\prime}}\left(\mathbb{R}^{n-1}\right)\right\|^{q}\right)^{\frac{r_{n}}{q}} d x_{n}\right)^{\frac{1}{r_{n}}}
$$

To proceed we note that Lemma 2 also holds if the base of the exponential is shifted from 2 to $R_{n}>1$ ( ince $R_{n}$ is a power of 2 ). We use this version below with $\left.\theta:=\frac{p_{n}}{r_{n}} \in\right] 0,1\left[\right.$, and setting $s_{0}=\frac{1}{p_{n}}-\frac{1}{r_{n}}, s_{1}=-\frac{1}{r_{n}}$,

$$
\theta s_{0}+(1-\theta) s_{1}=\frac{\theta}{p_{n}}-\frac{1}{r_{n}}=0 .
$$

Applying this to the $\ell_{q}^{0}$-norm, the above integral is majorised by

$$
c\left(\int_{\mathbb{R}}\left(\sup _{j} R_{n}^{s_{0} j}\left\|f_{j}\left(\cdot, x_{n}\right) \mid L_{p^{\prime}}\right\|\right)^{\theta r_{n}} d x_{n}\right)^{\frac{1}{r n}}\left(\sup _{j, x_{n}} R_{n}^{-\frac{j}{r n}}\left\|f_{j}\left(\cdot, x_{n}\right) \mid L_{p^{\prime}}\right\|\right)^{1-\theta} .
$$

Using Proposition 4 on the $L_{\left(r^{\prime}, \infty\right)}$-norm, one has for the last factor

$$
(\ldots)^{1-\theta} \leq c^{\prime}\left(\sup _{j} R_{n}^{\frac{j}{p_{n}}-\frac{j}{r_{n}}}\left\|f_{j} \mid L_{\vec{p}}\right\|\right)^{1-\theta} \leq c^{\prime}\left\|\sup _{j} R_{n}^{\frac{j}{p_{n}}-\frac{j}{r_{n}}}\left|f_{j}\right| \mid L_{\vec{p}}\right\|^{1-\theta}
$$

The first factor $(\ldots)^{\theta / p_{n}}$ can be treated similarly, and (39) holds.

Using Theorem 5 we arrive at the following Sobolev embedding. 
Theorem 6. Let $s, t \in \mathbb{R}, s>t$, and $\left.p_{j}, r_{j} \in\right] 0, \infty[$ fulfil

$$
s-\frac{1}{p_{1}}-\cdots-\frac{1}{p_{n}}=t-\frac{1}{r_{1}}-\cdots-\frac{1}{r_{n}}, \quad \forall k: r_{k} \geq p_{k} .
$$

There is then a continuous embedding $F_{\vec{p}, \infty}^{s}\left(\mathbb{R}^{n}\right) \hookrightarrow F_{\vec{r}, q}^{t}\left(\mathbb{R}^{n}\right)$ for every $\left.\left.q \in\right] 0, \infty\right]$.

Proof. Since the ball $B\left(0, A \cdot 2^{j}\right)$ is contained in the rectangle in (38) with $R_{k}=2$ for all $k$, it suffices to take a Littlewood-Paley decomposition of an arbitrary $u$ in $F_{\vec{p}, \infty}^{s}\left(\mathbb{R}^{n}\right)$ and then insert $f_{j}:=2^{t j} u_{j}$ into (39).

In the following section, the above embedding will be extended to a set-up with an additional anisotropy in $s$.

\section{Spaces with mixed norms and quasi-homogeneous smoothness}

As is well known, it is important, say for parabolic differential equations to consider spaces that are anisotropic in the quasi-homogeneous sense concerning the smoothness index $s$. This may be combined with the mixed Lebesgue norms in the way we now describe briefly.

Each coordinate $x_{j}$ in $\mathbb{R}^{n}$ is given a weight $a_{j} \geq 1$, and $\vec{a}=\left(a_{1}, \ldots, a_{n}\right)$; i.e. $\vec{a}=(1, \ldots, 1)$ is the case previously treated in this paper. With the anisotropic dilation $t^{\vec{a}} x:=\left(t^{a_{1}} x_{1}, \ldots, t^{a_{n}} x_{n}\right)$ for $t \geq 0$, and $t^{s \vec{a}} x:=\left(t^{s}\right)^{\vec{a}} x$ for $s \in \mathbb{R}$, and in particular $t^{-\vec{a}} x=\left(t^{-1}\right)^{\vec{a}} x$, the anisotropic distance function $|x|_{\vec{a}}$ is introduced as the unique $t>0$ such that $t^{-\vec{a}} x \in S^{n-1}$ (for $x \neq 0 ;|0|_{\vec{a}}=0$ ); i.e.

$$
\frac{x_{1}^{2}}{t^{2 a_{1}}}+\cdots+\frac{x_{n}^{2}}{t^{2 a_{n}}}=1
$$

For the reader's convenience we recall that $|\cdot|_{\vec{a}}$ is $C^{\infty}$ on $\mathbb{R}^{n} \backslash\{0\}$ by the Implicit Function Theorem; the formula $\left|t^{\vec{a}} x\right|_{\vec{a}}=t|x|_{\vec{a}}$ is seen directly, and this implies the triangle inequality:

$$
|x+y|_{\vec{a}} \leq|x|_{\vec{a}}+|y|_{\vec{a}}
$$

Indeed, it should be shown for $x \neq 0 \neq y$ that $1 \geq \sum_{j=1}^{n} \frac{\left(x_{j}+y_{j}\right)^{2}}{\left(|x|_{\vec{a}}+|y|_{\vec{a}}\right)^{2 a_{j}}}$, and since each fraction is invariant under $(x, y) \mapsto\left(t^{\vec{a}} x, t^{\vec{a}} y\right)$, it can be assumed that $|x|_{\vec{a}}+|y|_{\vec{a}}=1$. Then $0<|x| \leq|x|_{\vec{a}}<1$, for

$$
1=\sum_{j=1}^{n} x_{j}^{2}|x|_{\vec{a}}^{-2 a_{j}} \geq \sum_{j=1}^{n} x_{j}^{2}|x|_{\vec{a}}^{-2}=|x|^{2}|x|_{\vec{a}}^{-2} .
$$

With similar results for $y$, we get $|x+y| \leq|x|+|y| \leq|x|_{\vec{a}}+|y|_{\vec{a}} \leq 1$, whence the inequality after (46), as desired. 
As a preparation, we need the following analogues of the inequalities between the $\ell_{\infty}-, \ell_{2}$ - and $\ell_{1}$-norms on $\mathbb{C}^{n}$ :

$$
\max \left(\left|x_{1}\right|^{1 / a_{1}}, \ldots,\left|x_{n}\right|^{1 / a_{n}}\right) \leq|x|_{\vec{a}} \leq\left|x_{1}\right|^{1 / a_{1}}+\cdots+\left|x_{n}\right|^{1 / a_{n}} .
$$

The inequality to the right follows from (46), since $\left|\left(x_{1}, 0, \ldots\right)\right|_{\vec{a}}=\left|x_{1}\right|^{1 / a_{1}}$ etc. By taking $t$ equal to the above maximum, the left hand side of (45) would be $\geq 1$ (the maximum is attained), so that $t \leq|\xi| \vec{a}$.

Along with $|\cdot|_{\vec{a}}$, the Littlewood-Paley decomposition is chosen with the modification that $\Psi_{j}(\xi)=\psi\left(2^{-j}|\xi| \vec{a}\right)$. Then $\Phi_{j}$ is supported in the anisotropic corona $2^{j-1} \leq|\xi|_{\vec{a}} \leq 2^{j+1}$. As usual $u_{j}:=\mathcal{F}^{-1}\left(\Phi_{j} \hat{u}\right)$, but here it is understood that it is the anisotropic distance function $|\cdot|_{\vec{a}}$ that goes into the construction.

Using this, the anisotropic Lizorkin-Triebel space $F_{\vec{p}, q}^{s, \vec{a}}\left(\mathbb{R}^{n}\right)$, with $s \in \mathbb{R}$, $\vec{p} \in] 0, \infty\left[{ }^{n}\right.$ and $0<q \leq \infty$, consists of the $u$ in $\mathcal{S}^{\prime}\left(\mathbb{R}^{n}\right)$ having finite quasinorms

$$
\left\|u\left|F_{\vec{p}, q}^{s, \vec{a}}\|=\|\left(\sum_{j=0}^{\infty} 2^{s q j}\left|u_{j}(\cdot)\right|^{q}\right)^{\frac{1}{q}}\right| L_{\vec{p}}\right\|
$$

The corresponding Besov space $B_{\vec{p}, \infty}^{s, \vec{a}}\left(\mathbb{R}^{n}\right)$, now with $0<p_{k} \leq \infty$ for all $k$, is given by the quasi-norm

$$
\left\|u \mid B_{\vec{p}, q}^{s, \vec{a}}\right\|=\left(\sum_{j=0}^{\infty} 2^{s q j}\left\|u_{j} \mid L_{\vec{p}}\right\|^{q}\right)^{\frac{1}{q}} .
$$

These are more precisely anisotropic spaces of the quasi-homogeneous and mixed norm type. In the case of usual Lebesgue norms, i.e. $p_{1}=\cdots=$ $p_{n}=p$, such spaces have been used in general investigations, for example of microlocal properties of non-linear differential equations in [25] and of pointwise multiplication in [9]. For $1<p_{k}<\infty, k=1, \ldots, n$ and positive $s$, anisotropic Triebel-Lizorkin spaces based on mixed $L_{p}$-norms have been investigated in the second edition of the famous book of Besov, Il'in and Nikol'skij [2]. There they are introduced by means of differences. c.f. Remark 10 below.

Let us first note that $\| \cdot\left|F_{\vec{p}, q}^{s, \vec{a}}\right| \mid$ and $\| \cdot\left|B_{\vec{p}, q}^{s, \vec{a}}\right| \mid$ are quasi-norms. Indeed, (21) implies that for $\lambda=\min \left(1, q, p_{1}, \ldots, p_{n}\right)$ their powers $\|\cdot\|^{\lambda}$ are subadditive ; i.e. an analogue of (21) holds for them. So by taking $\ell_{1 / \lambda}$ and $\ell_{(1 / \lambda)^{*}-\text { norms, }}$ $\left(\frac{1}{\lambda}\right)^{*}=\frac{1}{1-\lambda}$, the quasi-triangle inequality results:

$$
\|f+g\| \leq\left(\|f\|^{\lambda}+\|g\|^{\lambda}\right)^{1 / \lambda} \leq 2^{1-\lambda}(\|f\|+\|g\|) .
$$

When both $q \geq 1$ and all $p_{k} \geq 1$, the spaces are therefore Banach spaces (in view of the completeness shown in Proposition 9 below). 
As usual the simple embeddings $F_{\vec{p}, q}^{s, \vec{a}} \hookrightarrow F_{\vec{p}, q}^{s-\varepsilon, \vec{a}}$ for $\varepsilon>0$, respectively $F_{\vec{p}, q}^{s, \vec{a}} \hookrightarrow F_{\vec{p}, r}^{s, \vec{a}}$ for $q<r$ are easy to see. Similarly for $B_{\vec{p}, q}^{s, \vec{a}}$. A direct argument and the generalised Minkowski inequality gives that

$$
B_{\vec{p}, \min \left(p_{1}, \ldots, p_{n}\right)}^{s, \vec{a}} \hookrightarrow F_{\vec{p}, q}^{s, \vec{a}} \hookrightarrow B_{\vec{p}, \max \left(p_{1}, \ldots, p_{n}\right)}^{s, \vec{a}} .
$$

Next we show that the Sobolev embedding for these spaces is a direct consequence of the previous inequality for distributions fulfilling the geometric rectangle condition (38).

Theorem 7. Let $s, t \in \mathbb{R}, s>t$, and $\left.\left.p_{k}, r_{k} \in\right] 0, \infty\right]$ fulfil

$$
s-\frac{a_{1}}{p_{1}}-\cdots-\frac{a_{n}}{p_{n}}=t-\frac{a_{1}}{r_{1}}-\cdots-\frac{a_{1}}{r_{n}}, \quad \forall k: r_{k} \geq p_{k} .
$$

For any $q \in] 0, \infty]$ there are then continuous embeddings $F_{\vec{p}, \infty}^{s, \vec{a}} \hookrightarrow F_{\vec{r}, q}^{t, \vec{a}}$ (all $\left.r_{k}<\infty\right)$ and $B_{\vec{p}, q}^{s, \vec{a}} \hookrightarrow B_{\vec{r}, q}^{t, \vec{a}}$ between these spaces over $\mathbb{R}^{n}$.

Proof. Since $\operatorname{supp} \Phi_{j}$ is contained in the set where $|\xi|_{\vec{a}} \leq 2^{j+1}$, the left inequality in (48) gives for all $k=1, \ldots, n$ that $\left|\xi_{k}\right| \leq 2^{a_{k}+j a_{k}}$. Hence $\operatorname{supp} \Phi_{j}$ is a subset of the rectangle in (38) for $R_{k}=2^{a_{k}}$ and $A=2^{\max \left(a_{1}, \ldots, a_{n}\right)}$.

Applying Theorem 5 to $f_{j}:=2^{t j} u_{j}$ for an arbitrary $u$ in $F_{\vec{p}, \infty}^{s, \vec{a}}\left(\mathbb{R}^{n}\right)$ therefore yields that $\left\|u\left|F_{\vec{r}, q}^{t, \vec{a}}\|\leq c\| u\right| F_{\vec{p}, \infty}^{s, \vec{a}}\right\|$ under the assumption in (53). The $B$-case follows from (26).

Remark 8. In [2, Thm. 29.10] embeddings of $F$-spaces into $L_{\vec{r}}$ and into Besov spaces are studied. Our Theorem 7 supplements and improves (at least partly) the assertions stated there.

Finally, as a supplement to the existing literature, we add the next result. It is another application of the mixed-norm version of the Nikol' skij-Plancherel-Polya inequality, cf. Proposition 4 above.

Proposition 9. For $s \in \mathbb{R}, 0<p_{k}<\infty(1 \leq k \leq n)$ and $0<q \leq \infty$ the Lizorkin-Triebel space $F_{\vec{p}, q}^{s, \vec{a}}\left(\mathbb{R}^{n}\right)$ is a quasi-Banach space with continuous embeddings

$$
\mathcal{S}\left(\mathbb{R}^{n}\right) \hookrightarrow F_{\vec{p}, q}^{s, \vec{a}}\left(\mathbb{R}^{n}\right) \hookrightarrow \mathcal{S}^{\prime}\left(\mathbb{R}^{n}\right)
$$

Analogous results hold for the $B_{\vec{p}, q}^{s, \vec{a}}$ spaces with $\left.\left.p_{k} \in\right] 0, \infty\right]$ for all $k$.

Proof. By (52), it suffices to show (54) in the Besov case; and $q=\infty$ is also enough. That $\mathcal{S}\left(\mathbb{R}^{n}\right) \hookrightarrow B_{\vec{p}, \infty}^{s, \vec{a}}\left(\mathbb{R}^{n}\right)$ is a direct consequence of the definition, with a proof similar to the isotropic one in $[18,2.3 .3]$.

The continuity of $B_{\vec{p}, \infty}^{s, \vec{a}}\left(\mathbb{R}^{n}\right) \hookrightarrow \mathcal{S}^{\prime}\left(\mathbb{R}^{n}\right)$ can also be carried over from [18, 2.3.3]. In so doing, the anisotropies can be handled by Proposition 4 (like in the proof of 
Theorem 7), which for any $u \in B_{\vec{p}, \infty}^{s, \vec{a}}\left(\mathbb{R}^{n}\right)$ gives

$$
\left\|u_{j}\left|L_{\infty}\left\|\leq c 2^{j\left(\frac{a_{1}}{p_{1}}+\cdots+\frac{a_{n}}{p_{n}}\right)}\right\| u_{j}\right| L_{\vec{p}}\right\|
$$

Then one can set $\tilde{\Phi}_{j}=\Phi_{j-1}+\Phi_{j}+\Phi_{j+1}$, so that $\tilde{\Phi}_{j} \equiv 1$ on $\operatorname{supp} \Phi_{j}$; this gives for $u \in B_{\vec{p}, \infty}^{s, \vec{a}}$ and $\psi \in \mathcal{S}\left(\mathbb{R}^{n}\right)$

$$
\begin{aligned}
|\langle u, \bar{\psi}\rangle| & \leq \sum_{j=0}^{\infty}\left|\left\langle u, \overline{\mathcal{F}^{-1}\left(\Phi_{j} \tilde{\Phi}_{j} \hat{\psi}\right)}\right\rangle\right| \\
& \leq \sum_{j=0}^{\infty} c 2^{j\left(\frac{a_{1}}{p_{1}}+\cdots+\frac{a_{n}}{p_{n}}\right)}\left\|u_{j}\left|L_{\vec{p}}\|\| \mathcal{F}^{-1}\left(\tilde{\Phi}_{j} \hat{\psi}\right)\right| L_{1}\right\| \\
& \leq c^{\prime}\left\|u\left|B_{\vec{p}, \infty}^{s, \vec{a}}\left\|\sum_{j=0}^{\infty} 2^{\left(-s+\frac{a_{1}}{p_{1}}+\cdots+\frac{a_{n}}{p_{n}}\right) j}\right\| \mathcal{F}^{-1}\left(\tilde{\Phi}_{j} \hat{\psi}\right)\right| L_{1}\right\| .
\end{aligned}
$$

Formally the last infinite series has the structure of a $B_{1,1}^{\frac{a_{1}}{p_{1}}+\cdots+\frac{a_{n}}{p_{n}}-s}$-norm on $\psi$. However, the fact that the family $\left(\tilde{\Phi}_{j}\right)$ appears instead of the decomposition $\left(\Phi_{j}\right)$ is inconsequential, for the proof of the continuity of $\mathcal{S} \hookrightarrow B_{p, q}^{s}$ also gives an estimate in this situation. So for some seminorm $p_{N}$ on $\mathcal{S}$ it holds that $|\langle u, \bar{\psi}\rangle| \leq c^{\prime \prime}\left\|u \mid B_{\vec{p}, \infty}^{s, \vec{a}}\right\| p_{N}(\psi)$, as desired.

Completeness is shown for the $F$-case $([18,2.3 .3]$ is without details on this). Given a fundamental sequence $\left(u_{l}\right)$ in $F_{\vec{p}, q}^{s, \vec{a}}$, the just shown continuity gives that $u_{l}-u_{m}$ belongs eventually to any given neighbourhood of 0 in $\mathcal{S}^{\prime}\left(\mathbb{R}^{n}\right)$; hence $\left(u_{l}\right)$ converges in $\mathcal{S}^{\prime}$ to some $u$. So $\check{\Phi}_{j} * u_{l} \rightarrow \check{\Phi}_{j} * u$ in $\mathcal{S}^{\prime}$ for $l \rightarrow \infty$ (since the index $l$ refers to the sequence, we shall write $\breve{\Phi}_{j} * u_{l}$ for the frequency modulations by means of the Littlewood-Paley decomposition). Hence $\check{\Phi}_{j} * u_{l}(x)=\left\langle u_{l}, \check{\Phi}_{j}(x-\cdot)\right\rangle$ converges pointwisely to $\check{\Phi}_{j} * u(x)$.

It remains to prove that $u \in F_{\vec{p}, q}^{s, \vec{a}}\left(\mathbb{R}^{n}\right)$ and $u_{l} \rightarrow u$ in the topology of this space. For $q<\infty$ the sum over $j \in \mathbb{N}_{0}$ can be seen as an integration w.r.t. the counting measure, and then $n+1$ applications of Fatou's lemma give $\left\|u\left|F_{\vec{p}, q}^{s, \vec{a}}\|\leq \liminf \| u_{l}\right| F_{\vec{p}, q}^{s, \vec{a}}\right\|<\infty$ (it is convenient that positive measurable functions always have integrals in $[0, \infty]$ and that Tonelli's theorem implies the measurability during the successive integrations). Given $\varepsilon>0$ and $N$ so that $\left\|u_{m}-u_{l} \mid F_{\vec{p}, q}^{s, \vec{a}}\right\|<\varepsilon$ for $m, l>N$, similar applications of Fatou's lemma gives

$$
\begin{aligned}
\left\|u-u_{l} \mid F_{\vec{p}, q}^{s, \vec{a}}\right\| & \leq\left\|\left(\liminf _{m} \sum_{j} 2^{s j q}\left|\check{\Phi}_{j} *\left(u_{m}-u_{l}\right)\right|^{q}\right)^{1 / q} \mid L_{\vec{p}}\right\| \\
& \leq \limsup _{m}\left\|u_{m}-u_{l} \mid F_{\vec{p}, q}^{s, \vec{a}}\right\|<\varepsilon
\end{aligned}
$$

This shows the convergence in $F_{\vec{p}, q}^{s, \vec{a}}$. For $q=\infty$ it is seen directly that $\sup _{j}\left|\check{\Phi}_{j} * u(x)\right| \leq \liminf _{l}\left(\sup _{j}\left|\check{\Phi}_{j} * u_{l}(x)\right|\right)$, and thence the conclusions follow as 
above. In the $B$-cases the ingredients are the same, only with the $j$-integration carried out last.

Remark 10. To conclude we comment on the background.

Anisotropic Sobolev (or Bessel potential) spaces $H_{p}^{s, \vec{a}}$ and Besov spaces $B_{p, q}^{s, \vec{a}}$ (with $1<p<\infty$ respectively $1 \leq p, q \leq \infty$, partly with $s>0$ ) and in particular embedding relations between them have been investigated e.g. in the monographs of Nikol'skij [11] and Besov, Il'in and Nikol'skij [1], [2]. Nikol'skij and his coauthors departed from a definition based on derivatives and differences. For a

characterization of anisotropic Lizorkin-Triebel spaces $F_{\vec{p}, q}^{s, \vec{a}}$ by differences we refer to Yamazaki [24, Thm. 4.1] and Seeger [13] (for $\vec{p}=(p, \ldots, p)$, but general $\vec{a}$ ). Since Sobolev spaces $H_{p}^{s, \vec{a}}$ represent particular cases of the Lizorkin-Triebel scale, there is some overlap between our work and these quoted books. In connection with anisotropic Lizorkin-Triebel spaces we would like to mention also Triebel [16] and Stöckert, Triebel [15] for the Fourier-analytic characterization; and concerning the $\varphi$-transform and characterization by atoms, we refer to Dintelmann [5] and Farkas [6].

As others before us, we have preferred to define the anisotropy in terms of the function $|\cdot|_{\vec{a}}$. This procedure is well known and goes back at least to the 1960's. A list of some basic properties of $|\cdot|_{\vec{a}}$ can be found in [23], together with further historical remarks.

One advantage of using $|\cdot|_{\vec{a}}$ is that it gives an efficient formalism where the powerful tools from Fourier analysis and distribution theory are easy to invoke. This is clearly illustrated by the rather manageable proofs of e.g. Theorems 5 and 7. In general the Fourier-analytic approach gives streamlined, if not simpler proofs of some basic properties of the spaces.

Finally we note that this paper has been partly motivated by some works of Weidemaier [21, 22] and Denk, Hieber and Prüess [4], whose results on traces in connection with parabolic problems can be roughly summarised as follows: taking traces by setting $x_{1}=0$ in the anisotropic $F_{\vec{p}, q}^{s, \vec{a}}$-spaces, with $s=2$ and $\vec{a}=(1, \ldots, 1,2)$, leads to trace spaces in the same scale, and only to Besov spaces if $p_{1}=\cdots=p_{n}$.

Concerning the trace problem for the full scale $F_{\vec{p}, q}^{s, \vec{a}}$, i.e. with general $s, \vec{a}, \vec{p}$ and $q$, we have corroborated this conclusion in another joint work [10]. With the present article our intention was to extract some preparations that should be of independent interest.

\section{References}

[1] O.V. Besov, V.P. Ilin, and S.M. Nikol'skij, Integral Representations of Functions and Imbedding Theorems, V. H. Winston \& Sons, Washington, 
D.C., 1978-79, Translated from the Russian, Scripta Series in Mathematics, Edited by Mitchell H. Taibleson.

[2] Integral Representations of Functions and Imbedding Theorems, Nauka, Moscow, 1996, 2nd edition (in russian).

[3] H. Brezis and P. Mironescu, Gagliardo-Nirenberg, composition and products in fractional Sobolev spaces, J. Evol. Equ., 1 (2001), 387-404.

[4] R. Denk, M. Hieber, and J. Prüess, Optimal $L_{p}-L_{q}$-regularity for parabolic problems with inhomogeneous boundary data, Preprint, University of Konstanz, Germany, No. 205, 2005.

[5] P. Dintelmann, Fourier multipliers between weighted anisotropic function spaces: Besov-Triebel spaces. Z. Anal. Anwend., 15 (1996), 579-601.

[6] W. Farkas, Atomic and subatomic decompositions in anisotropic function spaces. Math. Nachr., 209 (2000), 83-113.

[7] L. Hörmander, The Analysis of Linear Partial Differential Operators, Grundlehren der mathematischen Wissenschaften, Springer Verlag, Berlin, 1983, 1985.

[8] B. Jawerth, Some observations on Besov and Lizorkin-Triebel spaces, Math. Scand., 40 (1977), 94-104.

[9] J. Johnsen, Pointwise multiplication of Besov and Triebel-Lizorkin spaces, Math. Nachr., 175 (1995), 85-133.

[10] J. Johnsen and W. Sickel, On the trace problem for Lizorkin-Triebel spaces with mixed norms, Preprint R-2006-31, Aalborg University, 2006 (submitted).

[11] S. M. Nikol'skij, Approximation of Functions of Several Variables and Embedding Theorems, Springer, 1975.

[12] H.-J. Schmeisser and H. Triebel, Topics in Fourier Analysis and Function Spaces, Geest and Portig, 1987, Wiley, Chichester 1987.

[13] A. Seeger, A note on Triebel-Lizorkin spaces. Banach Center Publ., PWN Polish Sci. Publ. Warsaw, 22(1989), 391-400.

[14] B. Stöckert, Ungleichungen vom Plancherel-Pólya-Nikolskŭ-Typ in gewichteten $L_{p}^{\Omega}$-Räumen mit gemischten Normen, Math. Nachr., 86 (1978), 19-32.

[15] B. Stöckert and H. Triebel, Decomposition methods for function spaces of $B_{p, q}^{s}$ and $F_{p, q}^{s}$ type, Math. Nachr., 89 (1979), 247-267.

[16] H. Triebel, Fourier Analysis and Function Spaces, Teubner-Texte zur Mathematik, vol. 7, Teubner Verlagsgesellschaft, Leipzig, 1977.

[17] H. Triebel, Spaces of Besov-Hardy-Sobolev Type, Teubner-Texte zur Mathematik, vol. 15, Teubner Verlagsgesellschaft, Leipzig, 1978. 
[18] _ Theory of function Spaces, Monographs in Mathematics, vol. 78, Birkhäuser Verlag, Basel, 1983.

[19] A. P. Unin'skij, Embedding theorems for a class of functions with mixed norm, Dokl. Akad. Nauk. SSSR 166 (1966), 806-808.

[20] - Inequalities in a mixed norm for trigonometric polynomials and entire functions of finite order, Embedding theorems and applications. Nauka, Moskva, (1970), 212-218.

[21] P. Weidemaier, Existence results in $L_{p}-L_{q}$ spaces for second order parabolic equations with inhomogeneous Dirichlet boundary conditions, Progress in partial differential equations, Vol. 2 (Pont-à-Mousson, 1997), Pitman Res. Notes Math. Ser., vol. 384, Longman, Harlow, 1998, pp. 189-200.

[22] —, Maximal regularity for parabolic equations with inhomogeneous boundary conditions in Sobolev spaces with mixed $L_{p}$-norm, Electron. Res. Announc. Amer. Math. Soc., 8 (2002), 47-51 (electronic).

[23] M. Yamazaki, A quasi-homogeneous version of paradifferential operators, $I$. Boundedness on spaces of Besov type, J. Fac. Sci. Univ. Tokyo Sect. IA, Math., 33 (1986), 131-174.

[24] — A quasi-homogeneous version of paradifferential operators, II. A symbol calculus, J. Fac. Sci. Univ. Tokyo Sect. IA, Math., 33 (1986), 311345.

[25] _ A quasi-homogeneous version of the microlocal analysis for nonlinear partial differential equations, Japan. J. Math., 14 (1988), 225-260.

Department of Mathematical Sciences

Aalborg University, Fredrik Bajers Vej 7G

DK-9220 Aalborg Øst, Denmark

(E-mail : jjohnsen@math.aau.dk)

Mathematics Department

Friedrich-Schiller-University Jena

Ernst-Abbe-Platz 2, D-07743 Jena, Germany

(E-mail : sickel@minet.uni-jena.de) 


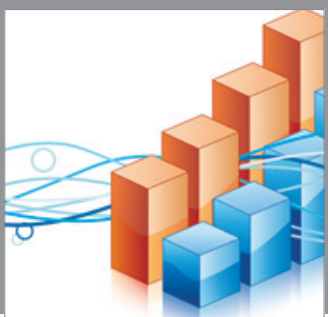

Advances in

Operations Research

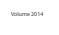

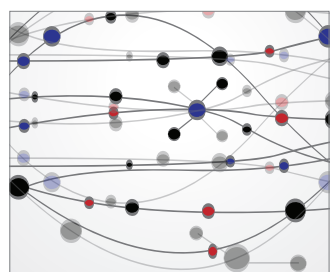

\section{The Scientific} World Journal
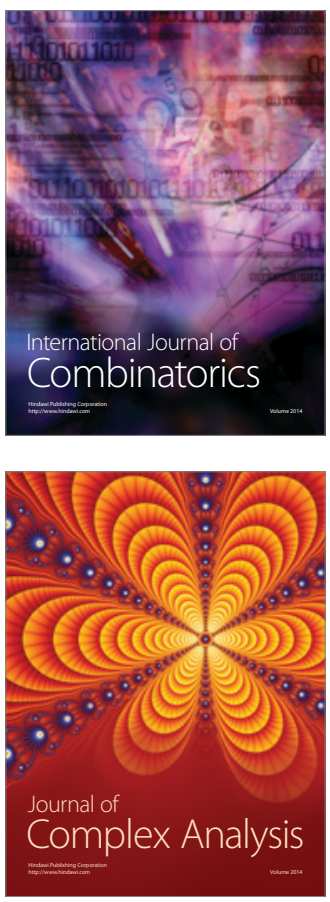

International Journal of

Mathematics and

Mathematical

Sciences
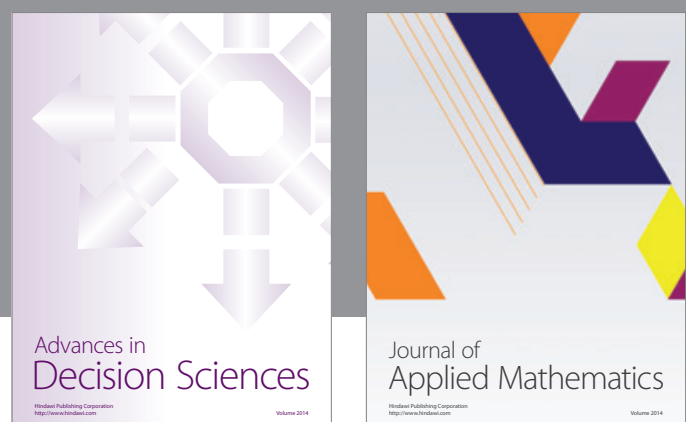

Journal of

Applied Mathematics
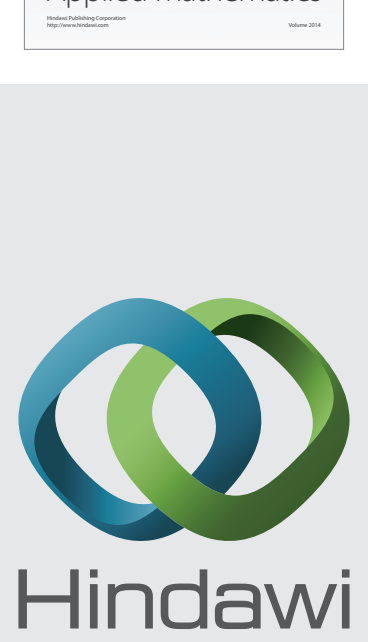

Submit your manuscripts at http://www.hindawi.com
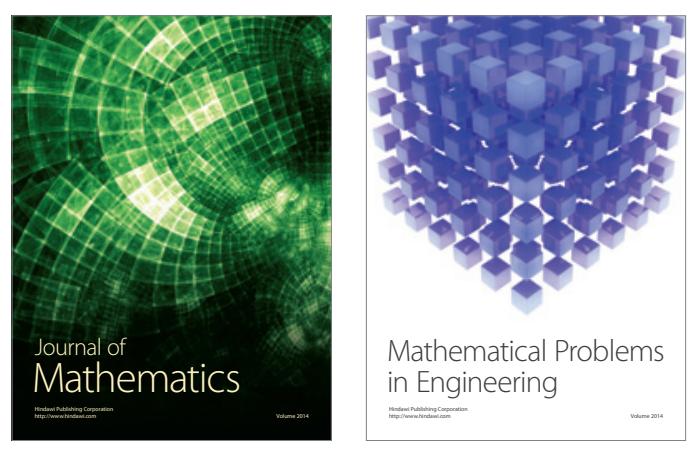

Mathematical Problems in Engineering
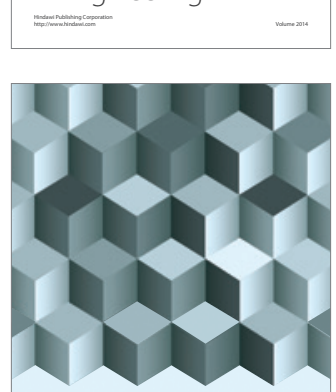

Journal of

Function Spaces
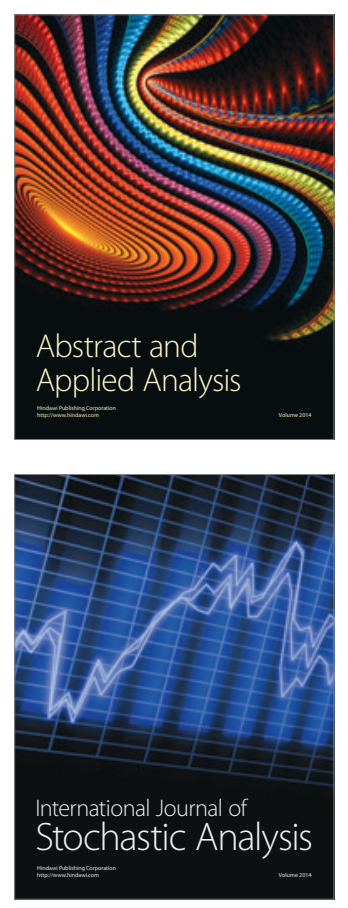

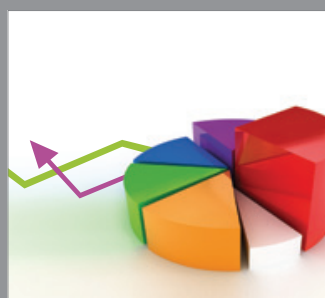

ournal of

Probability and Statistics

Promensencen
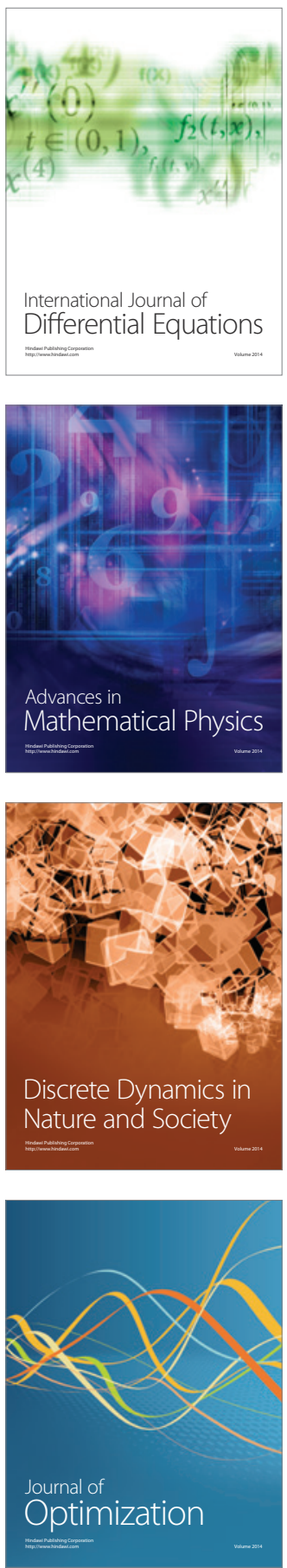